\title{
Generalization of the Einstein relation for single trajectories in deterministic subdiffusion
}

\author{
Takuma Akimoto* \\ Department of Mechanical Engineering, Keio University, Yokohama 223-8522, Japan \\ (Received 28 September 2011; revised manuscript received 2 December 2011; published 8 February 2012)

\begin{abstract}
Diffusion coefficients are intrinsically random in subdiffusion attributable to power-law trapping. Using deterministic biased and unbiased diffusion models, we investigate the Einstein relation for single trajectories in subdiffusion. The difference in the generalized Lyapunov exponent between biased and unbiased deterministic diffusions is related to the velocity under a bias. By Hopf's ergodic theorem, the ratios between the velocities and the Lyapunov exponents for single trajectories converge to a universal constant, which is proportional to the strength of the bias. Based on a certain transport coefficient obtained from a single trajectory, we provide a relation for the transport coefficients divided by the Lyapunov exponent and generalize the Einstein relation for single trajectories.
\end{abstract}

DOI: 10.1103/PhysRevE.85.021110

PACS number(s): 05.60.Cd, 02.50.-r, 05.45.Ac

\section{INTRODUCTION}

The intrinsic randomness of time-averaged observables is of great interest in nonequilibrium statistical mechanics. Equilibrium systems are characterized by a few macroscopic observables, which are time averages of microscopic observation functions. Macroscopic observables fluctuate about their ensemble averages in an equilibrium state, meaning that the time averages of the microscopic observable functions converge to their ensemble averages. In contrast, macroscopic observables such as the diffusion coefficient and fluorescence intensity do not converge to constants, but show large fluctuations in nonequilibrium (nonsteady) phenomena such as anomalous diffusions and intermittent processes [1-4]. In particular, diffusion coefficients of biological molecules in cells [1-3] and the fluorescence times in single nanocrystals [4] show large fluctuations, indicating ergodicity breaking.

The intrinsic randomness of time-averaged observables is universal in models characterized by power-law trapping time distributions with divergence means [5-10]. A typical example is the continuous time random walk (CTRW), which is a random walk with a random continuous trapping time. In CTRWs with infinite mean trapping times, the diffusion coefficient shows intrinsic randomness $[8,10]$. Although the time average is not equal to the ensemble average for such systems (i.e., ergodicity breaking), time averages converge in distribution, this behavior being called distributional ergodicity [11].

In dynamical systems, distributional ergodicity refers to ergodicity in infinite measure systems because dynamical systems related to stochastic models with infinite mean trapping times have infinite invariant measures [12]. In infinite measure systems, the time average of an observation function converges in distribution. This distribution is determined by an infinite invariant measure as well as the properties of the

\footnotetext{
*akimoto@z8.keio.jp
}

Published by the American Physical Society under the terms of the Creative Commons Attribution 3.0 License. Further distribution of this work must maintain attribution to the author(s) and the published article's title, journal citation, and DOI. observation function [7]. For an infinite measure-preserving transformation $T$, the distribution of the time average of an $L^{1}(\mu)$ function $f(x)$ (i.e., $\left.\int|f| d \mu<\infty\right)$ converges to the Mittag-Leffler distribution [13]

$$
\frac{1}{a_{n}} \sum_{k=0}^{n-1} f \circ T^{k} \Rightarrow M_{\alpha},
$$

provided that $\int f d \mu \neq 0$, where $M_{\alpha}$ is a random variable with a Mittag-Leffler distribution of order $\alpha$ and $\Rightarrow$ signifies convergence in distribution. The sequence $a_{n}$, called the return sequence, is related to the nonstationary underlying process. In deterministic subdiffusion, where the mean square displacement grows sublinearly $\left\langle x_{n}^{2}\right\rangle \propto n^{\alpha}(0<\alpha<1)$, the scaling of $a_{n}$ is the same as $\left\langle x_{n}^{2}\right\rangle$ [9].

In diffusion processes, an external constant force $F$ generates a drift. In general biased random walks with anomalous diffusion, the Einstein relation

$$
V=\frac{F D}{2 k_{B} T}
$$

holds for a small force $F D / k_{B} T \ll 1[14,15]$, where $V$ is the velocity under a bias, $D$ is the diffusion coefficient under no bias, $k_{B}$ is the Boltzmann constant, and $T$ is the temperature. Diffusion behavior in hyperbolic chaotic dynamical systems is well known. Using the escape rate formula, one can show that the diffusion coefficient is represented by the difference between the Lyapunov exponent and the Kolmogorov-Sinai entropy [16]. In a Lorentz gas, the largest Lyapunov exponent is suppressed by an external field and there is a relation between the Lyapunov exponents and a bias $[17,18]$. However, little is known about the relation between dynamical instability and transport under a bias. Moreover, the Einstein relation for the transport coefficients obtained by single trajectories does not exist because the diffusion coefficient is intrinsically random in subdiffusion as a consequence of power-law trapping.

In this paper, we consider dynamical systems related to biased and unbiased CTRWs. Using the Lyapunov exponent, we obtain a relation between the macroscopic transport coefficient and the microscopic chaos in a deterministic subdiffusion. In particular, we relate the ensemble-averaged velocity to the difference in the generalized Lyapunov exponents between biased and unbiased dynamical systems. Using Hopf's ratio 
ergodic theorem, we show that the ratio between a timeaveraged observable and the Lyapunov exponent converges to a universal constant. Finally, we demonstrate a relation for the transport coefficients divided by the Lyapunov exponent. Even though the diffusion coefficient and velocity are intrinsically random, this relation provides the Einstein relation for single trajectories.

\section{DETERMINISTIC BIASED AND UNBIASED DIFFUSION MODELS}

Deterministic diffusion models can be represented by a one-dimensional map $T(x)$ with translational symmetry, $T(x+L)=T(x)+L(L=0, \pm 1, \ldots$,$) [19]. Under this$ symmetry, the map $T(x)$ can be reduced to a map on the unit interval $[-1 / 2,1 / 2], R(x)$ [20]. Here we construct the reduced map based on the CTRW. The CTRW is defined by a jump-length distribution $l(x)$ and a trapping-time distribution $\psi(t)$. For simplicity, we assume that the jump-length distribution is written by $l(x)=p \delta(x-1)+q \delta(x+1)(p+q=$ 1). Since trapping times in CTRWs are independently and identically distributed random variables, we can construct a one-dimensional piecewise linear intermittent map $R(x)$ where the distribution of the trapping time on $[-1 / 4,1 / 4]$ has the same scaling as in the CTRW [21].

We consider the following intermittent map as the reduced map (see Fig. 1)

$$
R(x ; z, c)= \begin{cases}\frac{x-3 / 4+c}{2 c-1 / 2} & x \in\left[-\frac{1}{2},-\frac{3}{4}+c\right), \\ \frac{x-3 / 4+c}{1-2 c} & x \in\left[-\frac{3}{4}+c,-\frac{1}{4}\right), \\ x-4^{z-1}(-x)^{z} & x \in\left[-\frac{1}{4}, 0\right), \\ x+4^{z-1} x^{z} & x \in\left[0, \frac{1}{4}\right), \\ \frac{x-c}{2 c-1 / 2} & x \in\left[\frac{1}{4}, c\right), \\ \frac{x-c}{1-2 c} & x \in[c, 1 / 2] .\end{cases}
$$

In what follows, we use the notation $R(x)$ instead of $R(x ; z, c)$ for brevity. The invariant measure corresponding to $R(x)$ is given by $d \mu_{c}=h_{c}(x)|x|^{1-z} d x$, where $h_{c}(x)$ is a continuous function [22]; this measure becomes infinite if $z \geqslant 2$. The asymptotic scaling of the distribution of the trapping time on $[-1 / 4,1 / 4]$ is given by $\psi(t) \propto t^{-1-\alpha}(t \rightarrow \infty)$ with $\alpha=$
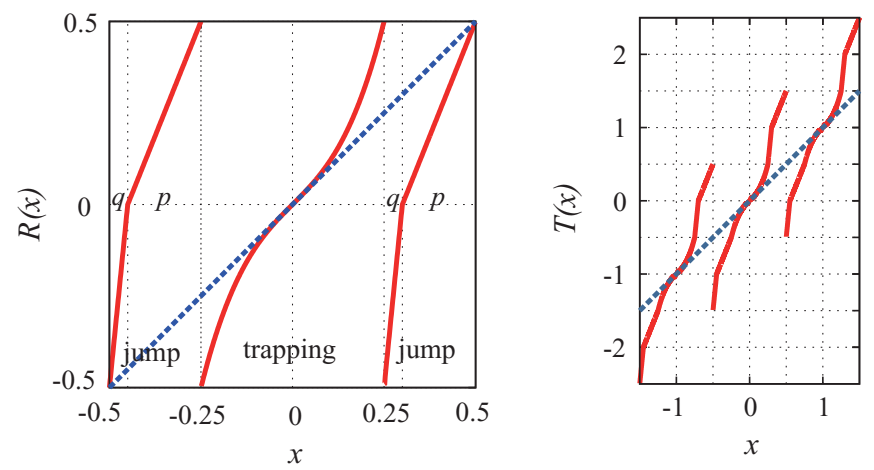

FIG. 1. (Color online) Reduced map $R(x)$ and the corresponding original map $T(x)[z=3.0$ and $c=0.3$ in Eq. (3)]. The solid curves represent those maps. $R(x)=x$ and $T(x)=x$ are represented by dashed lines for reference.

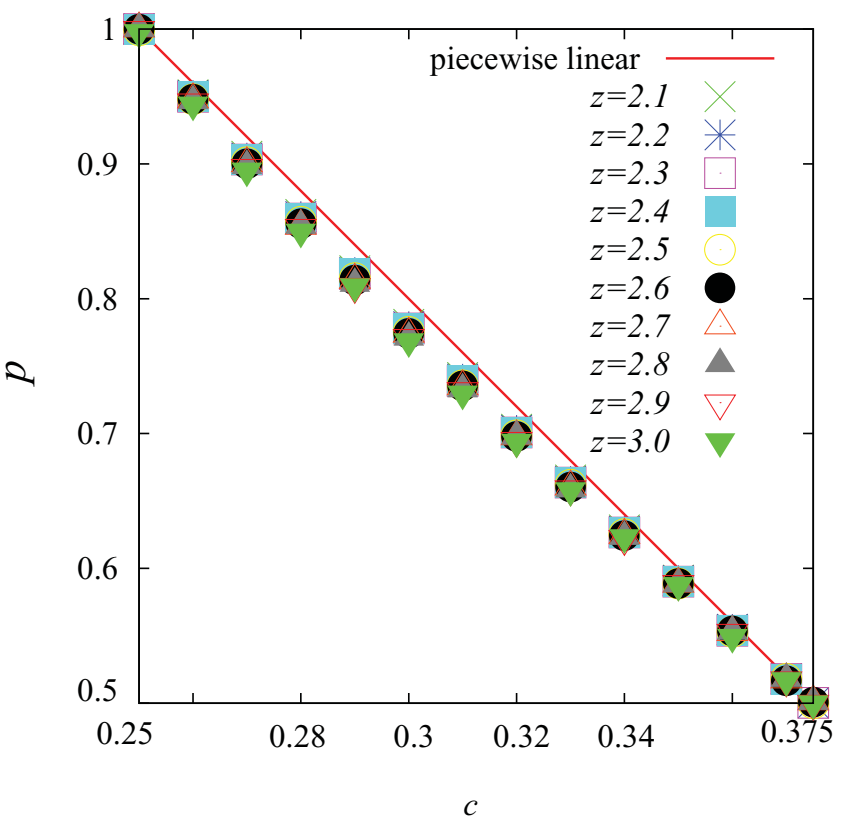

FIG. 2. (Color online) Probability $p$ for a right jump. The straight line represents $p=2-4 c$. Although $p$ does not satisfy $p=2-4 c$, $p \propto 2-4 c$ for small bias at different values of $z$. It follows that $p-q=C(3-8 c)$ for $|c-0.375| \ll 1(C<1)$.

$1 /(z-1)$. A bias is characterized by a point $c$, at which the slope of the map changes. If the reduced map is piecewise linear, the injection on the interval $[1 / 4,1 / 2]$ is uniform. Therefore, the probability for a right jump $p$ is given by the length of the interval $[c, 1 / 2]$. Although this fact does not hold in the intermittent reduced map (3), the relation between $p$ and $c$ is similar to that in the piecewise linear map (see Fig. 2). We note that, whereas the scaling exponents of the trapping-time distributions on $[0,1 / 4]$ and $[-1 / 4,0]$ are the same, the distribution on $[0,1 / 4]$ is slightly different from that on $[-1 / 4,0]$ because the derivatives $R(x)$ at $x=c+0$ and $c-0$ are different in biased models. ${ }^{1}$ If the mean trapping time diverges $(0<\alpha<1), R(x)$ has an infinite invariant measure [12] and the map $T(x)$ generates subdiffusion (i.e., $\left.\left\langle x_{n}^{2}\right\rangle \propto n^{\alpha}[19]\right)$.

\section{EINSTEIN RELATION}

The Einstein relation relates the velocity under bias to the diffusion coefficient under no bias. We focus on the Einstein relation for single trajectories. The time-averaged drift and mean square displacement are defined by

$$
\overline{\delta x_{m}}\left(N ; x_{0}\right) \equiv \frac{1}{N} \sum_{k=0}^{N-1}\left(x_{k+m}-x_{k}\right)
$$

\footnotetext{
${ }^{1}$ Strictly speaking, the trapping-time distribution is different from each biased dynamical system. However, the tails of the trapping-time distribution are exactly the same. Therefore, this difference does not affect a bias, whereas it affects the mean of the total number of jumps $\left\langle N_{m}\right\rangle$ slightly.
} 
and

$$
\overline{\delta x_{m}^{2}}\left(N ; x_{0}\right) \equiv \frac{1}{N} \sum_{k=0}^{N-1}\left(x_{k+m}-x_{k}\right)^{2}
$$

respectively. Since the observation function of a drift $f_{m}(x) \equiv$ $T^{m}(x)-x$ is an $L^{1}(\mu)$ function and $\int_{-1 / 2}^{1 / 2} f_{m}(x) d \mu \neq 0$ for $c \neq 0.375$, the distributional limit theorem, Eq. (1), holds. In particular, the distribution of the normalized time average of $f_{m}(x)$ converges to a Mittag-Leffler distribution $\frac{1}{a_{n}} \sum_{k=0}^{n-1} f_{m} \circ T^{k} \Rightarrow M_{\alpha}$, where the return sequence is given by

$$
a_{n} \propto \begin{cases}\frac{n}{\log n} & (z=2), \\ n^{\alpha} & (z>2) .\end{cases}
$$

Unlike the ensemble-averaged mean square displacement, the time-averaged mean square displacement shows normal diffusion even if $\alpha<1 \quad(z>2)$ [9]. Therefore, the time-averaged diffusion coefficient is defined by $\bar{D} \equiv$ $\overline{\delta x_{m}^{2}}\left(N ; x_{0}\right) / m$. According to Eq. (1), the time-averaged diffusion coefficient is intrinsically random for $z \geqslant 2$ [9]. With finite measures $(z<2)$, the time-averaged diffusion coefficient is equal to its ensemble average. The ensemble-averaged drift $\left\langle\delta x_{m}\right\rangle_{F}$ is given by $\left\langle\delta x_{m}\right\rangle_{F} \equiv\left\langle x_{m}-x_{0}\right\rangle_{F}$, where $\langle\cdot\rangle_{F}$ indicates the ensemble average under bias. Let $N_{m}$ be the total number of jumps until time $m$. In CTRWs, the ensemble-averaged drift and mean square displacement are given by $\left\langle\delta x_{m}\right\rangle_{F}=(p-$ $q)\left\langle N_{m}\right\rangle_{F}$ and $\left\langle\delta x_{m}^{2}\right\rangle_{0}=\left\langle N_{m}\right\rangle_{0}$ for $p=q$, respectively, where $\langle\cdot\rangle_{0}$ is the ensemble average under no bias. If the injection onto the interval $[-1 / 2,-1 / 4) \cup(1 / 4,1 / 2]$ is uniform, we have $p=2-4 c$ and $q=4 c-1$. Here, we assume that $\left\langle\delta x_{m}\right\rangle_{F}=A(p-q)\left\langle N_{m}\right\rangle_{F},\left\langle\delta x_{m}^{2}\right\rangle_{0}=B\left\langle N_{m}\right\rangle_{0}$ for $p=q$, and $p-q=C(3-8 c)$ for $|p-q| \ll 1$, where $A, B$, and $C$ are constants that do not depend on a bias $c$ for a small bias. These assumptions were confirmed by numerical simulations; the probability $p$ is shown in Fig. 2. In normal diffusion $\left\langle N_{m}\right\rangle_{F}$ is given by $\mu_{c}\left(J^{c}\right) m$, where $\mu_{c}$ is an invariant measure of $R(x)$ and $J^{c}$ is the complement of $J=[-1 / 4,1 / 4]$. In subdiffusion, $\left\langle N_{m}\right\rangle_{F} \propto m^{\alpha}$. We then have

$$
\left\langle\overline{\delta x_{m}}(N)\right\rangle_{F}=A(p-q) \sum_{k=0}^{N-1} \frac{\left\langle N_{k+m}\right\rangle_{F}-\left\langle N_{k}\right\rangle_{F}}{N} \cong\langle\bar{V}\rangle_{F} m,
$$

where we define the time-averaged velocity by $\bar{V} \equiv$ $\overline{\delta x_{m}}(N) / m$, which does not depend on $m$.

Let $p=W_{0} e^{a F / 2 k_{B} T}, q=W_{0} e^{-a F / 2 k_{B} T}$, where $W_{0}=1 /$ $\left(e^{a F / 2 k_{B} T}+e^{-a F / 2 k_{B} T}\right)$ and $a$ is a lattice spacing [14], and $\Delta n(k)$ is the total number of jumps in the interval $[k, k+m]$. Using $\left\langle\delta x_{m}\right\rangle_{F}=A(p-q)\left\langle N_{m}\right\rangle_{F}$, we have $\left\langle x_{k+m}-x_{k}\right\rangle_{F} \cong$ $A(p-q)\langle\Delta n(k)\rangle_{F}$. Here, we assume $\langle\Delta n(k)\rangle_{F} \cong\langle\Delta n(k)\rangle_{0}$. This assumption was also confirmed in numerical simulations, although these are not shown here. It follows that $\left\langle x_{k+m}-x_{k}\right\rangle_{F} \cong A(p-q)\langle\Delta n(k)\rangle_{0} \cong A(p-q)$ $\left\langle\left(x_{k+m}-x_{k}\right)^{2}\right\rangle_{0} / B$. We have

$$
\langle\bar{V}\rangle_{F} \cong \frac{A}{B}\langle\bar{D}\rangle_{0} C \varepsilon
$$

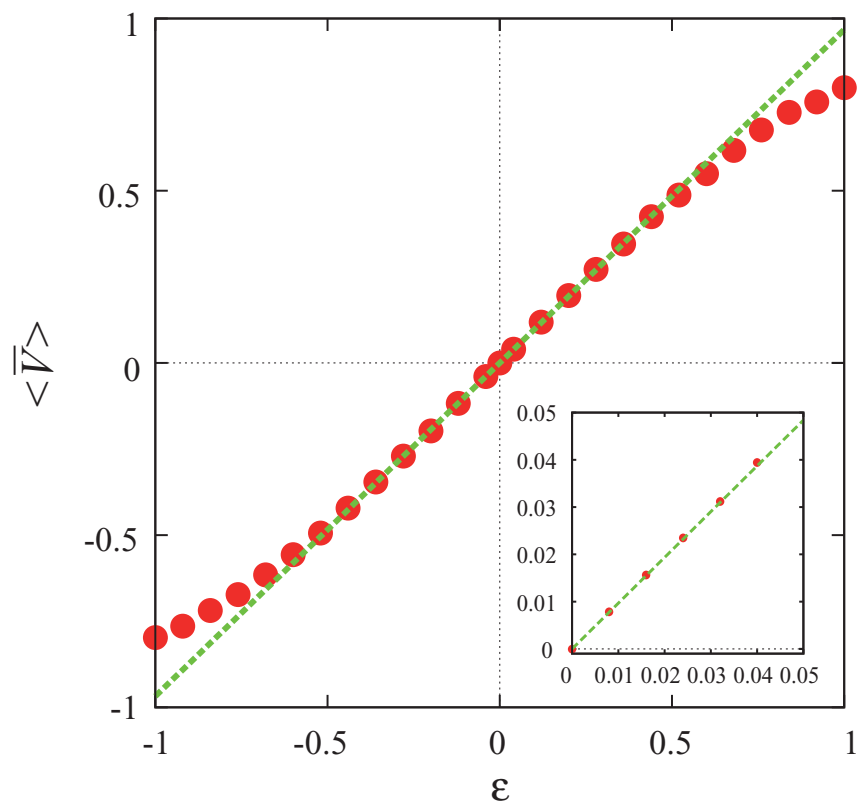

FIG. 3. (Color online) Einstein relation $\left(z=2.5\right.$ and $\left.N=10^{7}\right)$. Solid circles show the results of numerical simulations. The dashed line shows the theoretical results given by Eq. (8). Constants $\langle\bar{D}\rangle_{0}, A$, and $B$ are obtained by numerical simulations $\left(\langle\bar{D}\rangle_{0}=0.864\right.$ and $A C / B \cong 1.12$ ). The inset shows an amplified view of the region corresponding to small positive $\varepsilon$.

where $\varepsilon=3-8 c$. Thus, we obtain the Einstein relation

$$
\langle\bar{V}\rangle_{F}=\frac{F\langle\bar{D}\rangle_{0}}{2 k_{B} T}, \quad F \rightarrow 0,
$$

where $a=B / A$. Dissimilar to CTRWs, the parameter $a$ in our model is not equal to unity. This is because the constants $A$ and $B$ are distinct from unity because of stagnant motion near the indifferent fixed points; in other words, $A=B=1$ if the motion near these fixed points are completely stopped and the length of jumps is one as in a CTRW. Moreover, the relation $\langle\Delta n(k)\rangle_{F}=\langle\Delta n(k)\rangle_{0}$ holds in biased CTRWs.

We have confirmed the Einstein relation (9) by numerical simulations, as can be seen in Fig. 3. We note that the ensemble average in the Einstein relation is essential in subdiffusion because the time-averaged transport coefficients $\bar{D}$ and $\bar{V}$ are intrinsically random. If the time-averaged velocity $\bar{V}$ and the time-averaged diffusion coefficient $\bar{D}$ are calculated by independent and different initial conditions, $\bar{V} / \bar{D}$ becomes random, namely, the effective temperature $F \bar{D} / 2 k_{B} \bar{V}$ becomes random [23]. Figure 4 shows that the effective temperatures are random variables with a broad distribution.

\section{DYNAMICAL INSTABILITY AND TRANSPORT COEFFICIENT}

A dynamical instability is measured by the Lyapunov exponent, defined by

$$
\bar{\lambda}\left(N, x_{0}\right) \equiv \frac{1}{N} \sum_{k=0}^{N-1} \ln \left|T^{\prime}\left(x_{k}\right)\right| .
$$




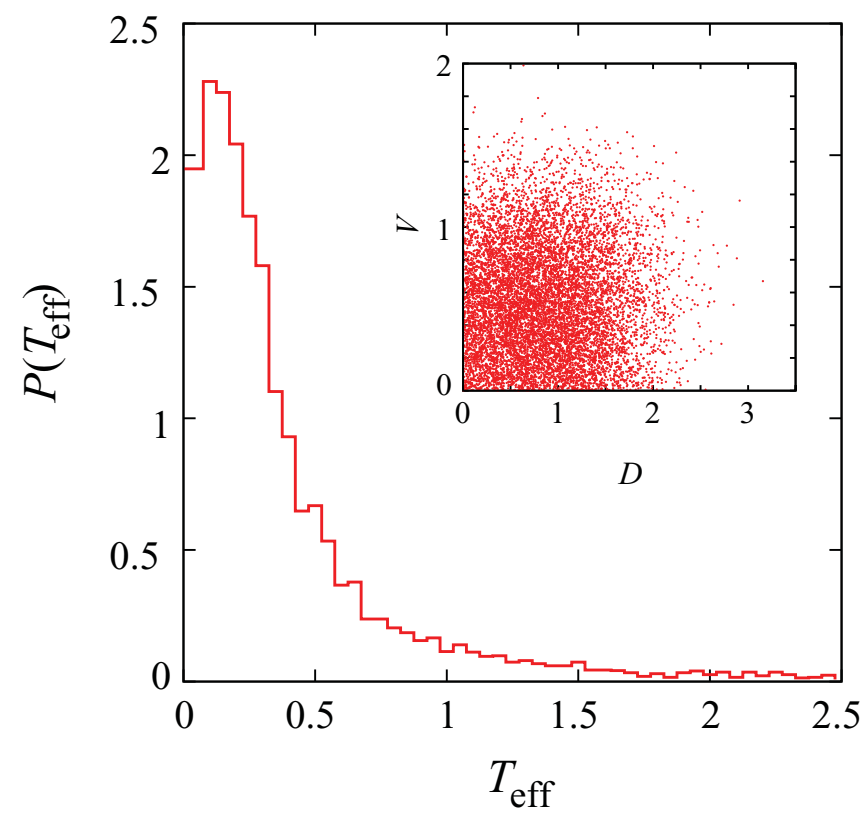

FIG. 4. (Color online) Probability density function of the normalized effective temperature $T_{\text {eff }} \equiv t_{\text {eff }} /\left\langle t_{\text {eff }}\right\rangle\left(z=2.5\right.$ and $\left.N=10^{7}\right)$, where $t_{\text {eff }} \equiv \bar{V} / \bar{D} \cdot \bar{V}$ for $c=0.3$ and $\bar{D}$ for $c=0.375$ are calculated independently. The inset shows $(\bar{D}, \bar{V})$ for different trajectories.

By Hopf's ergodic theorem [13]

$$
\frac{\sum_{k=0}^{n-1} f_{m}\left(T^{k} x\right)}{\sum_{k=0}^{n-1} g_{c}\left(T^{k} x\right)} \rightarrow C_{m}=\frac{\int_{0}^{1 / 2} f_{m} d \mu}{\int_{0}^{1 / 2} g_{c} d \mu}
$$

holds for almost all initial points, where $\underline{g}_{c}(x)=\ln \left|T^{\prime}(x)\right|$. Therefore, the time-averaged velocity $\bar{V}(N ; x)$ and the Lyapunov exponent $\bar{\lambda}(N ; x)$ satisfy the following relation, $\bar{V}(N ; x) / \bar{\lambda}(N ; x) \rightarrow C_{m} / m$ as $N \rightarrow \infty$ for almost all $x$. Since $\overline{\delta x_{m}}(N) \propto m$ for a large $m, C_{m} / m$ does not depend on $m$. Let $C_{m} / m$ equal $\chi_{V}$, we then obtain a relation between the velocity and the Lyapunov exponent for almost all initial points $x$

$$
\frac{\bar{V}(N ; x)}{\bar{\lambda}(N ; x)} \rightarrow \chi_{V} \quad \text { as } \quad N \rightarrow \infty .
$$

As shown in Fig. 5, there exists a universal constant $\chi_{V}$ obtained by Hopf's ergodic theorem. In general, the ensemble average of the Lyapunov exponent decreases with increasing the velocity (see Fig. 6). Nevertheless, the time-averaged velocity is proportional to the Lyapunov exponent. The constant $\chi_{V}$ is a universal constant that does not depend on the initial point. Using the ensemble average and Eq. (8), we obtain

$$
\chi_{V}=\frac{\langle\bar{V}\rangle_{F}}{\langle\bar{\lambda}\rangle_{F}}=\frac{A K\langle\bar{D}\rangle_{0}}{B\langle\bar{\lambda}\rangle_{F}} C \varepsilon
$$

We consider the difference in the generalized Lyapunov exponent between an unbiased and a biased dynamical system. The generalized Lyapunov exponent is the ensemble average of the normalized Lyapunov exponent $[12,24]$

$$
\Lambda \equiv\left\langle\lim _{n \rightarrow \infty} \frac{1}{a_{n}} \sum_{k=0}^{n-1} g_{c}\left(x_{k}\right)\right\rangle,
$$

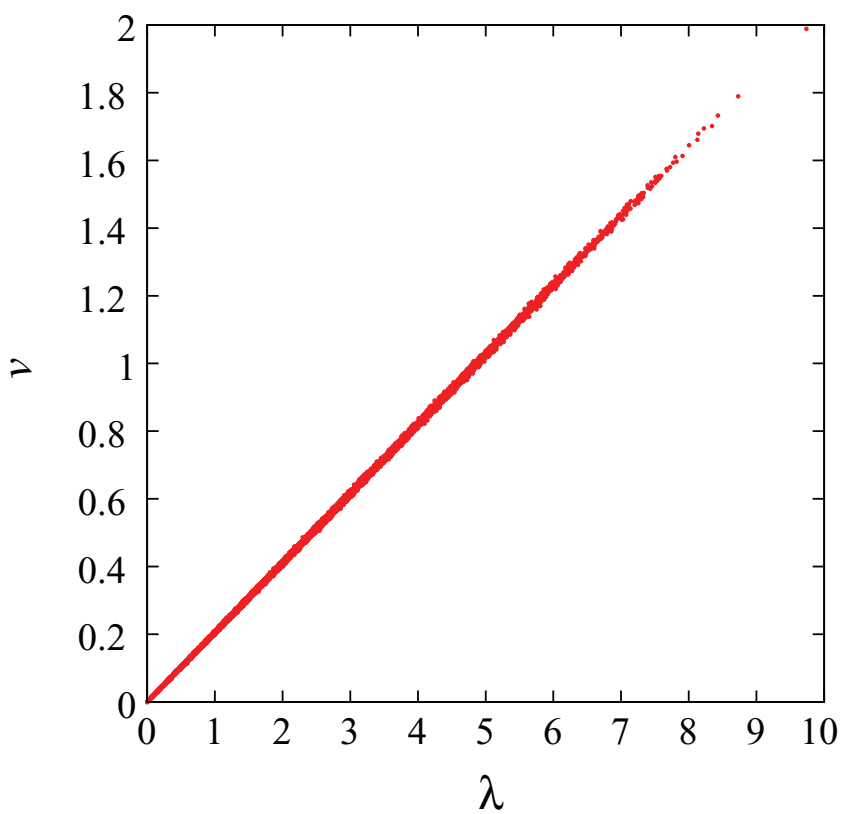

FIG. 5. (Color online) The normalized time-averaged velocity $v \equiv N \bar{V} / N^{\alpha}$ vs. the normalized Lyapunov exponent $\lambda \equiv N \bar{\lambda} / N^{\alpha}$ ( $z=2.5, c=0.3$, and $N=10^{7}$ ). Dots represent $(\lambda, v)$ for different trajectories. The linear relation between $\lambda$ and $v$ according to Hopf's ergodic theorem (12) holds.

where $\langle\cdot\rangle$ represents the ensemble average of the initial points $x_{0}$ and $a_{n}$ is the return sequence. Note that the initial density is absolutely continuous with respect to the Lebesgue measure and satisfies the condition $\left\langle g_{c}\left(x_{0}\right)\right\rangle<\infty$. The assumption $\langle\Delta n(k)\rangle_{F} \cong\langle\Delta n(k)\rangle_{0}$ means that the statistical property of the reinjection to the indifferent fixed points is almost the same. In other words, invariant measures for biased and unbiased maps are almost the same. That is, the generalized Lyapunov

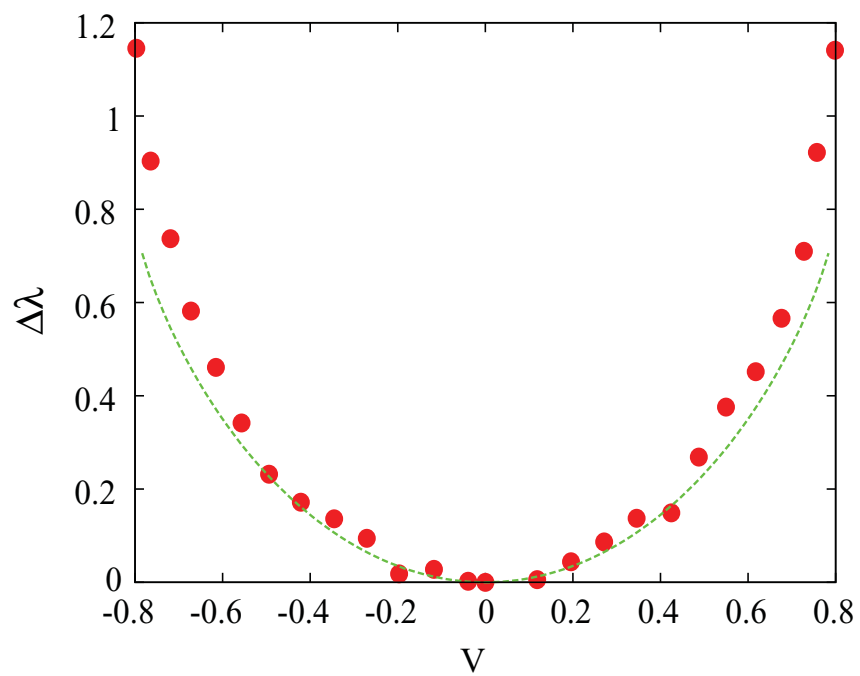

FIG. 6. (Color online) Relation between $\Delta \lambda$ and $V(z=2.5$ and $N=10^{7}$ ). Solid circles are the results of numerical simulations and the dashed line is the theoretical curve (20), where $V_{\max }$ and $\mu_{c_{0}}\left(J^{c}\right)$ are obtained by numerical simulations. In particular, we calculate $\mu_{c_{0}}\left(J^{c}\right)$ by $\lim _{n \rightarrow \infty}\left\langle\sum_{k=0}^{n} 1_{J^{c}}\left(R^{k} x\right) / a_{n}\right\rangle$, and we set $a_{n}=n^{\alpha}$. 
exponent restricted to $J=[-1 / 4,1 / 4]$ is almost the same. The difference in the generalized Lyapunov exponent defined by $\Delta \lambda=n\left(\langle\bar{\lambda}(x)\rangle_{0}-\langle\bar{\lambda}(x)\rangle_{F}\right) / a_{n}$ is given by

$$
\Delta \lambda=\frac{1}{a_{n}} \sum_{k=0}^{n-1}\left(\left\langle g_{c_{0}}\left(x_{k}\right) 1_{J^{c}}\left(x_{k}\right)\right\rangle_{0}-\left\langle g_{c}\left(x_{k}\right) 1_{J^{c}}\left(x_{k}\right)\right\rangle_{F}\right),
$$

where $c_{0}=0.375$. Thus,

$$
\Delta \lambda=\int_{J^{c}} g_{c_{0}}(x) d \mu_{c_{0}}-\int_{J^{c}} g_{c}(x) d \mu_{c} \cong \Delta \lambda_{J^{c}} \mu_{c_{0}}\left(J^{c}\right),
$$

where $\mu_{c_{0}}$ and $\mu_{c}$ are invariant measures for biased and unbiased maps, respectively, and $\Delta \lambda_{J^{c}}$ is the difference in the generalized Lyapunov exponent restricted to $J^{c}$. If the injection to $J^{c}$ is uniform, we have

$$
\frac{\int_{J^{c}} g_{c_{0}}(x) d \mu_{c_{0}}}{\mu_{c_{0}}\left(J^{c}\right)}=2 \int_{J^{c}} g_{c_{0}}(x) d x=\ln 4
$$

and

$$
\frac{\int_{J^{c}} g_{c}(x) d \mu_{c}}{\mu_{c_{0}}\left(J^{c}\right)}=2 \int_{J^{c}} g_{c}(x) d x=q \ln \frac{2}{q}+p \ln \frac{2}{p} .
$$

Therefore, we have

$$
\Delta \lambda_{J^{c}}=\ln 2+p \ln p+(1-p) \ln (1-p) .
$$

The normalized velocity and its maximum are defined as $V \equiv \lim _{n \rightarrow \infty}\left\langle\delta x_{n}\right\rangle_{F} / n^{\alpha}$ and $V_{\max } \equiv \lim _{n \rightarrow \infty}\left\langle N_{n}\right\rangle_{F} / n^{\alpha}$, respectively. We use $\frac{V}{V_{\max }}=p-q$. Then, the probability $p$ can be expressed in terms of $V$ and $V_{\max }: p=\left(1+V / V_{\max }\right) / 2$. Using $V$ and $V_{\max }$, we obtain a relation between the difference between the generalized Lyapunov exponent and the normalized velocity

$$
\Delta \lambda=\frac{\mu_{c_{0}}\left(J^{c}\right)}{2} S\left(\frac{V}{V_{\max }}\right),
$$

where $S(x)=(1+x) \ln (1+x)+(1-x) \ln (1-x)$. By numerical simulations, we have confirmed that this relation (20) is also valid except for large $V$ (Fig. 6). The generalized Lyapunov exponent has maximum at $V=0$ and decreases with increasing $|V|$.

\section{GENERALIZED EINSTEIN RELATION FOR SINGLE TRAJECTORIES}

For a small bias, the difference in the Lyapunov exponent for biased and unbiased deterministic diffusion given by Eq. (20) is sufficiently small [i.e., $\Delta \lambda=O(\varepsilon)]$. By $\langle\bar{\lambda}\rangle_{F}=\langle\bar{\lambda}\rangle_{0}+O(\varepsilon)$, the constant $\chi_{V}$ is given by

$$
\chi_{V}=\frac{A\langle\bar{D}\rangle_{0}}{B\langle\bar{\lambda}\rangle_{0}} C \varepsilon+O\left(\varepsilon^{2}\right)
$$

Since $\bar{D} / \bar{\lambda}$ converges to a constant for almost all trajectories $\bar{D} / \bar{\lambda}=\langle\bar{D}\rangle_{0} /\langle\bar{\lambda}\rangle_{0} \equiv \chi_{D}^{0}$. Thus, we derive a relation between $\chi_{V}$ and $\chi_{D}^{0}$

$$
\chi_{V}=\frac{A \chi_{D}^{0}}{B} C \varepsilon, \quad|\varepsilon| \ll 1 .
$$

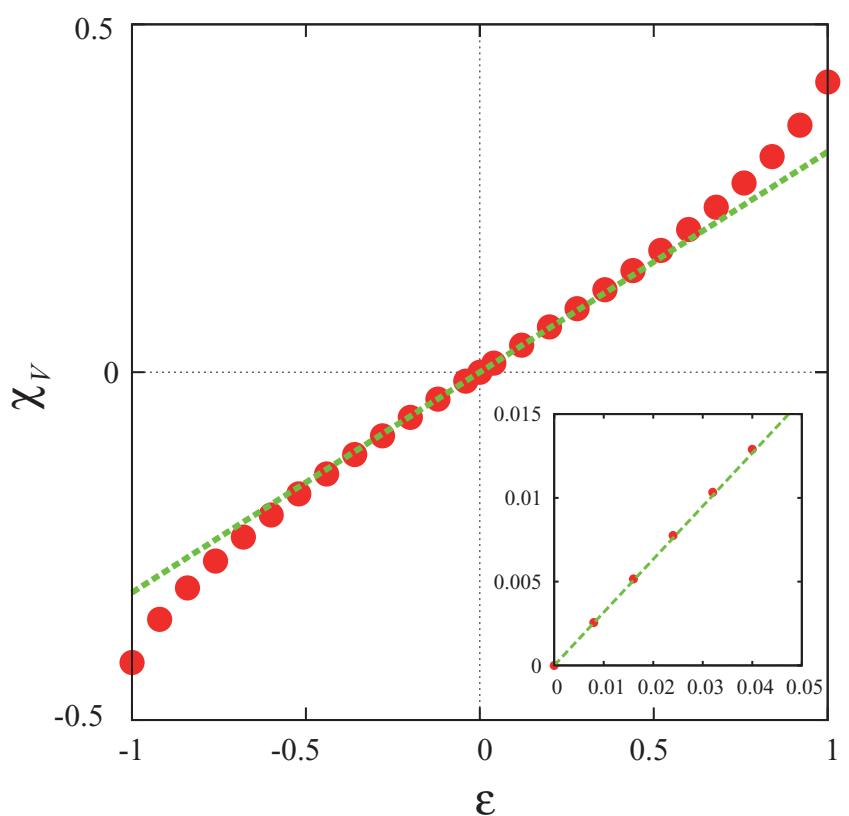

FIG. 7. (Color online) Generalized Einstein relation $(z=2.5$ and $N=10^{7}$ ). The solid circles show the results of numerical simulations; the dashed line shows the theoretical results given by Eq. (21). Constants $A, B,\langle\bar{D}\rangle_{0}$, and $\langle\bar{\lambda}\rangle_{0}$ are obtained by numerical simulations $\left(\langle\bar{D}\rangle_{0}=0.864,\langle\bar{\lambda}\rangle_{0}=3.05\right.$, and $\left.A C / B \cong 1.12\right)$. The inset is an amplification of the region for small positive $\varepsilon$.

Thus, the Einstein relation is generalized by recasting it in terms of the ratio of the time-averaged transport coefficients with the Lyapunov exponent, $\chi_{V}$ and $\chi_{D}^{0}$

$$
\chi_{V}=\frac{F \chi_{D}^{0}}{2 k_{B} T}, \quad F \rightarrow 0 .
$$

We note that this substitution is key in establishing constant transport coefficients. The above relation has been confirmed by numerical simulations, where the constants $\chi_{D}^{0}$ and $\chi_{V}$ are the ensemble-averaged values in Fig. 7. Hopf's ergodic theorem for $\chi_{D}^{0}$ has been also confirmed by numerical simulations.

\section{DISCUSSION}

We have generalized the Einstein relation by substituting the time-averaged transport coefficients with those divided by the Lyapunov exponent. We comment briefly on this generalized Einstein relation for single trajectories [i.e., Eq. (23)]. In subdiffusion due to a power-law trapping, it is impossible to obtain the Einstein relation based on the time average because time averages are intrinsically random. The remarkable point in our generalization is the above-mentioned substitution. In stochastic models such as CTRW, it is difficult to estimate the Lyapunov exponent. However, suitable time-averaged observables such as $N_{t} / t$ can help to re-express the transport coefficients so as to obtain a generalized version of the Einstein relation. For example, this relation can be expressed in the form

$$
\chi_{V}=\frac{F \chi_{D}^{0}}{2 k_{B} T}, \quad F \rightarrow 0,
$$


where $\chi_{V} \equiv \bar{V} /\left(N_{t} / t\right)$ and $\chi_{D}^{0} \equiv \bar{D} /\left(N_{t} / t\right)$. We note that $\chi_{V}$ and $\chi_{D}^{0}$ can be calculated from a single trajectory under bias and unbiased conditions, respectively.

\section{ACKNOWLEDGMENTS}

The author thanks T. Miyaguchi and C. P. Dettman for useful discussions and Grant-in-Aid for Young Scientists (B) (No. 22740262) for financial support.
[1] I. Golding and E. C. Cox, Phys. Rev. Lett. 96, 098102 (2006).

[2] A. Granéli, C. C. Yeykal, R. B. Robertson, and E. C. Greene, Proc. Natl. Acad. Sci. USA 103, 1221 (2006).

[3] J. Szymanski and M. Weiss, Phys. Rev. Lett. 103, 038102 (2009).

[4] X. Brokmann, J. P. Hermier, G. Messin, P. Desbiolles, J. P. Bouchaud, and M. Dahan, Phys. Rev. Lett. 90, 120601 (2003).

[5] G. Margolin and E. Barkai, Phys. Rev. Lett. 94, 080601 (2005).

[6] G. Margolin and E. Barkai, J. Stat. Phys. 122, 137 (2006).

[7] T. Akimoto, J. Stat. Phys. 132, 171 (2008).

[8] Y. He, S. Burov, R. Metzler, and E. Barkai, Phys. Rev. Lett. 101, 058101 (2008).

[9] T. Akimoto and T. Miyaguchi, Phys. Rev. E 82, 030102(R) (2010).

[10] T. Miyaguchi and T. Akimoto, Phys. Rev. E 83, 031926 (2011).

[11] T. Miyaguchi and T. Akimoto, Phys. Rev. E 83, 062101 (2011).

[12] T. Akimoto and Y. Aizawa, Chaos 20, 033110 (2011).
[13] J. Aaronson, An Introduction to Infinite Ergodic Theory (American Mathematical Society, Providence, RI, 1997).

[14] J. Bouchaud and A. Georges, Phys. Rep. 195, 127 (1990).

[15] E. Barkai and V. N. Fleurov, Phys. Rev. E 58, 1296 (1998).

[16] P. Gaspard and G. Nicolis, Phys. Rev. Lett. 65, 1693 (1990).

[17] C. P. Dettmann, G. P. Morriss, and L. Rondoni, Phys. Rev. E 52, 5746 (1995).

[18] G. P. Morriss, C. P. Dettmann, and D. J. Isbister, Phys. Rev. E 54, 4748 (1996).

[19] T. Geisel and S. Thomae, Phys. Rev. Lett. 52, 1936 (1984).

[20] G. Zumofen and J. Klafter, Phys. Rev. E 47, 851 (1993).

[21] T. Akimoto, T. Hasumi, and Y. Aizawa, Phys. Rev. E 81, 031133 (2010).

[22] M. Thaler, Isr. J. Math. 46, 67 (1983).

[23] Z. Shemer and E. Barkai, Phys. Rev. E 80, 031108 (2009).

[24] N. Korabel and E. Barkai, Phys. Rev. Lett. 102, 050601 (2009). 